\title{
Análise da Requalificação da Praça Miguel Rossafa, Umuarama - PR
}

\author{
Requalification Analysis of Miguel Rossafa Square, Umuarama - PR
}

Análisis de la Rehabilitación de la Plaza Miguel Rossafa, Umuarama - PR

Carolina Cardoso

Mestranda, UEM, Brasil

carolinac.arq@hotmail.com

Yara Campos Miranda

Mestranda, UEM, Brasil yaracamposmiranda1@gmail.com

Bruno Luiz Domingos De Angelis

Professor, Doutor, UEM, Brasil brucagen@uol.com.br 


\section{RESUMO}

As ruas e praças constituem os dois elementos fundamentais que estruturam a morfologia urbana das cidades. Em se tratando especialmente das praças, é necessário que estes espaços livres ofereçam um ambiente adequado para a realização de atividades e onde a população se sinta acolhida. Desta forma, o planejamento das cidades e de seus espaços livres apresenta um papel importante na manutenção da qualidade do espaço urbano, indicando as ações necessárias a fim de amparar as necessidades dos indivíduos. O objetivo deste artigo é analisar a evolução das estruturas de um espaço livre da cidade de Umuarama - PR. Para isto, foi realizado um estudo de caso na Praça Miguel Rossafa. O estudo é caracterizado pela análise da configuração da praça em dois momentos: em 2003, logo após a reforma de qualificação, e em 2017, obtendo, assim, um panorama das modificações graduais realizadas no espaço livre. O método utilizado para avaliação quantitativa e qualitativa da praça, proposto por De Angelis, Castro e Angelis Neto (2004), consiste na análise de suas estruturas, vegetação, morfologia e tipologia. Os resultados mostram que as reformas realizadas na praça, ainda que de forma gradual, melhoraram a sua pontuação. Entretanto, a falta de planejamento das requalificações urbanas oferece não só melhorias para a praça, mas também ocasiona novos problemas devido à falta de estudo da configuração do espaço livre.

PALAVRAS-CHAVE: Espaço livre. Morfologia. Qualidade ambiental.

\section{ABSTRACT}

The streets and the squares are the two key elements that structure the urban morphology of cities. In the case especially of the squares, it is necessary that these open spaces provide a suitable environment for performing activities where people feel welcomed. Thus the planning of cities and their open spaces presents an important role in maintaining the quality of urban space, indicating the necessary actions in order to support the needs of the individuals. The objective of this article is to analyze the structures' evolution of the open space in the city of Umuarama - PR. For this, a study case was carried out in Miguel Rossafa Square. The study is characterized by the analysis of the configuration in two moments: in 2003, soon after the qualification reform, and in 2017, thus obtaining an overview of the gradual modifications made in the open space. The method used for quantitative and qualitative evaluation of the square, proposed by De Angelis, Castro and Angelis Neto (2004), consists of the analysis of its structures, vegetation, morphology and typology. The results show that the reforms carried out in the square, although gradually, have improved their score. However, the lack of planning of urban requalifications not only offers improvements to the square, but also causes new problems due to the lack of study of the open space configuration.

KEYWORDS: Open space. Morphology. Environmental quality.

\section{RESUMO}

Las calles y plazas son los dos elementos clave que estructuran la morfología urbana de las ciudades. En el caso particular de las plazas, es necesario que estos espacios libres proporcionan un ambiente adecuado para la realización de actividades y donde las personas se sienta bienvenidas. Así, la planificación de las ciudades y sus áreas abiertas juega un papel importante en el mantenimiento de la calidad del espacio urbano, indicando las acciones necesarias con el fin de apoyar las necesidades de los individuos. El objetivo del artículo es analizar la evolución de las estructuras de un espacio libre en la ciudad de Umuarama - PR. Para esto, se realizó un estudio de caso en la Plaza Miguel Rossafa. El estudio se caracteriza por análisis de la configuración de la plaza en dos etapas: en 2003, después de la reforma de la calificación, y en 2017, obteniendo así una visión general de los cambios graduales realizados en el espacio libre. El método utilizado para la evaluación cuantitativa y cualitativa de la plaza, propuesta por De Angelis, Castro y Neto Angelis (2004), consiste en el análisis de sus estructuras, la vegetación, la morfología y tipología. Los resultados muestran que las reformas en la plaza, aunque de forma gradual, mejoraron su puntuación. Sin embargo, la falta de planificación urbana en la recalificación ofrece no sólo mejoras en la plaza, sino que también trae nuevos problemas debido a la falta de estudio de la configuración del espacio libre.

PALABRAS CLAVE: Espacio abierto. Morfología. Calidad del medio ambiente. 

A metodologia de avaliação quantitativa e qualitativa, proposta por De Angelis, Castro e Angelis Neto (2004), consiste na identificação da estrutura da praça, incluindo informações sobre os aspectos paisagísticos e dos elementos existentes neste espaço público, bem como o seu respectivo estado de manutenção e conservação. Segundo os autores, esta é uma metodologia que visa realizar o levantamento, cadastramento, diagnóstico e avaliação das praças, fundamentada em dois aspectos: estrutura física e utilização. Uma vez que o enfoque desta pesquisa é analisar apenas a estrutura da praça, alguns dos itens propostos pela metodologia, relacionados ao aspecto de utilização, foram descartados.

A metodologia propõe os seguintes itens:

Estudo do mobiliário, estruturas e similares: neste item, foram avaliados quantitativa e qualitativamente o mobiliário, objetos e demais elementos presentes na praça. Para isso, foram elaboradas duas fichas, as quais foram adaptadas para a realização desta pesquisa. Para cada um dos itens existentes na praça, foram atribuídas notas de 0,0 (zero) a 4,0 (quatro), conforme a escala: $0-\mid 0,5 \leftrightarrow$ péssimo; $0,5-\mid$ 1,5 $\leftrightarrow$ ruim; $1,5-\mid 2,5 \leftrightarrow$ regular; $2,5-\mid$ 3,5 $\leftrightarrow$ bom; e 3,5-4,0 $\leftrightarrow$ ótimo.

Levantamento quantitativo da vegetação: o segundo item proposto por esta metodologia é o levantamento da vegetação existente na praça. Para isto, foi realizada a contagem dos indivíduos arbóreos, enquanto que as espécies arbustivas e herbáceas tiveram suas áreas medidas. Os elementos foram identificados de acordo com o gênero e a espécie vegetal a qual pertence. A avaliação qualitativa da vegetação está incorporada ao item anterior.

Inserção da praça na trama urbana: existem três sistemas principais de criação de traçados urbanos, sendo eles: sistema retangular, sistema radial e sistema triangular (SITTE, 1992). Para o terceiro item da metodologia, foi identificado qual sistema de trama urbana foi utilizado para a criação da praça estudada e sua respectiva morfologia.

Tipologia: o quarto item apresenta a identificação da tipologia da praça. A metodologia propõe as seguintes categorias de praças: praça de igreja; praça de descanso e/ou recreação; praça de circulação; praça monumental; e praça de significação visual.

A metodologia propõe, ainda, dois itens: estudo da toponímia e um questionário de opinião dos usuários. Para esta pesquisa, optou-se por eliminar estes itens, uma vez que o objetivo é analisar a morfologia da praça e os itens propostos pela metodologia não condizem com o objetivo da pesquisa.

Para identificar a estrutura da Praça Miguel Rossafa em seus diversos estágios, analisou-se o desenho técnico referente ao projeto da reforma da praça em 2003, disponibilizado pela prefeitura de Umuarama (em arquivo vetorial, formato dwg). Para obter a versão atualizada da 

social, a remodelação da Praça Miguel Rossafa apresentou características que iam de encontro com os princípios vigentes.

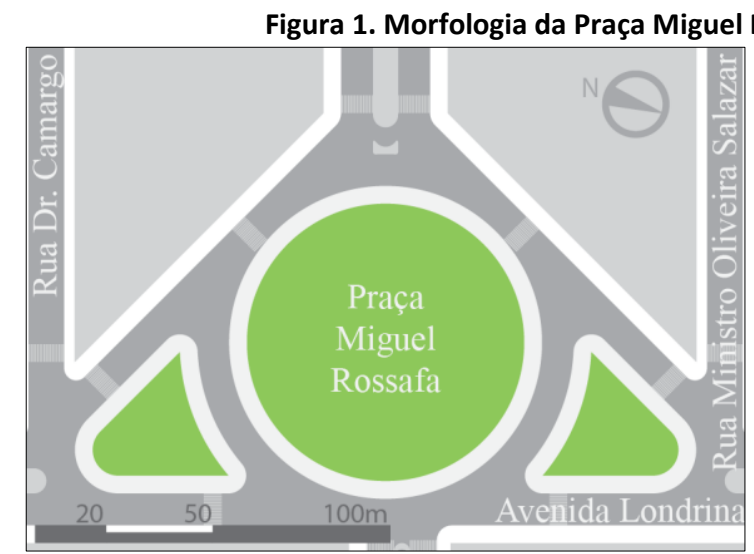

a) Morfologia antes da reforma de 2003

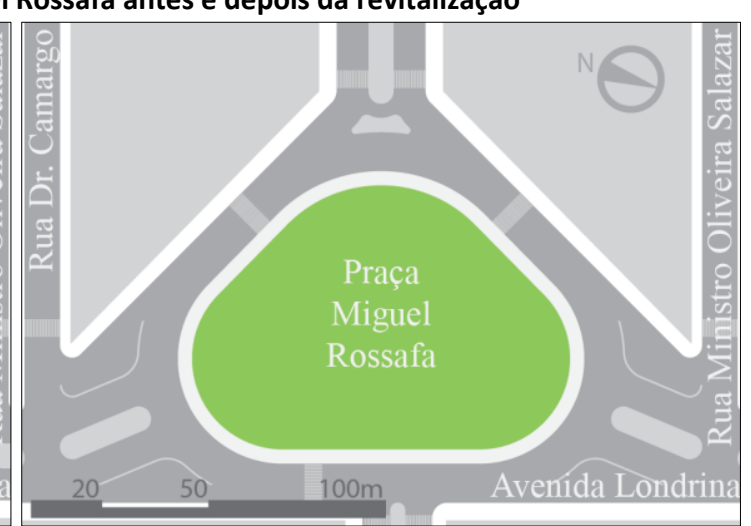

b) Morfologia depois da reforma de 2003 Fonte: PREFEITURA MUNICIPAL DE UMUARAMA (2003), modificado pelos autores.

No novo desenho arquitetônico (Figura 2a), os caminhos foram dispostos em eixos ortogonais dividindo a praça em seis setores, conformados por bancos em concreto. No centro da praça, construíram-se os sanitários e uma área de estar com mesas e bancos, também em concreto, cobertos por um pergolado circular. Os bancos e o pergolado apresentam características modernistas, o que causa desarmonia entre o desenho arquitetônico (de características ecléticas) e os elementos construídos. O mobiliário, além dos bancos e mesas fixos, comporta lixeiras, luminárias e um monumento. Ainda que na lei que permitiu a sua readequação esteja afirmando a preservação das árvores, as espécies de Tipuana tipu que se apresentavam bem desenvolvidas foram removidas (HULSMEYER, 2004). Inicialmente, nenhum equipamento de lazer ou cultural fora incorporado ao desenho arquitetônico da praça.

Com a consolidação da ocupação urbana na área da praça o fluxo viário se tornou intenso nessa região, prejudicando o acesso de pedestres ao espaço livre. Visando trazer mais segurança aos usuários, uma medida reformatória foi aplicada no espaço público no ano de 2011, onde se implantou faixas de pedestre elevadas em dois pontos do entorno da praça, a fim de forçar a redução da velocidade dos veículos.

Durante os anos de 2011 a 2015, novos elementos foram implantados na praça de forma pontual e gradual (Figura 2b). As mudanças realizadas no ano de 2011 incluem a alteração do paisagismo, que incorporou maciços de vegetação do tipo forração, a construção de uma fonte de água e uma academia da terceira idade (ATI). As flores dos maciços de vegetação são replantadas de acordo com o tempo de vida da espécie, e todas as mudas são provenientes de produção própria do município. Em 2012, foi construído um parque infantil. Pouco tempo depois, foi necessária a instalação de um gradil para impedir que as crianças se direcionassem 


\section{Revista Nacional de}

para a via de tráfego, pois esta apresentava riscos aos usuários. No ano de 2015 , toda a iluminação foi trocada e implantaram-se quadro elementos decorativos compostos por troncos de árvores reaproveitados e flores envazadas.

Figura 2. Desenho esquemático da requalificação da Praça Miguel Rossafa - 2003 e 2017

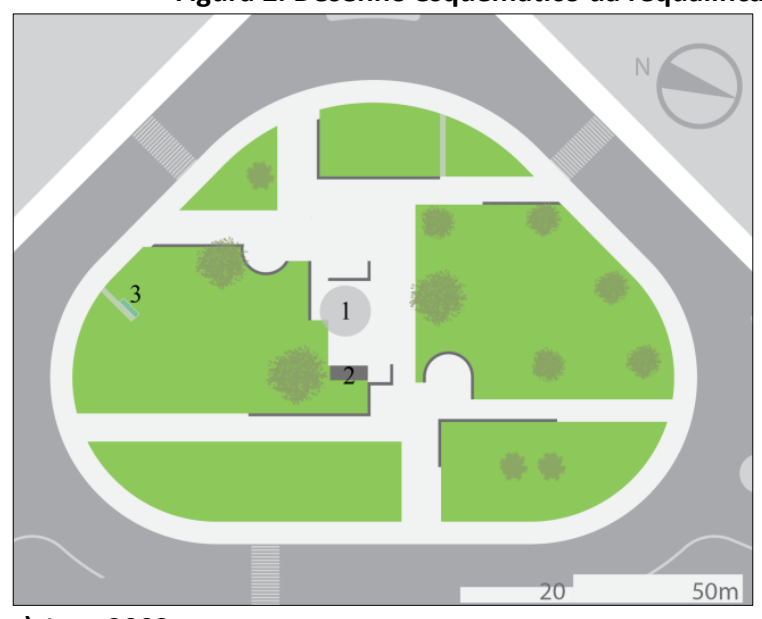

a) Ano: 2003

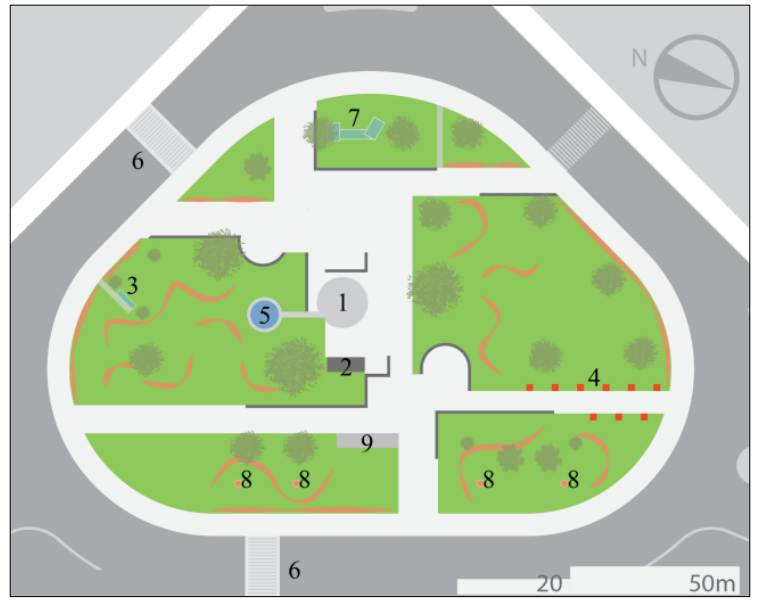

Legenda:

1) Pergolado com mesas

2) Sanitários

3) Monumento

4) Academia da Terceira Idade

5) Fonte de água

6) Faixas elevadas

7) Parque infantil

8) Decoração com troncos de árvores

9) Mastro para bandeiras

Canteiros com forração

b) Ano: 2017

Bancos em concreto

Fonte: PREFEITURA MUNICIPAL DE UMUARAMA (2003), modificado pelos autores.

Neste período de reformas pontuais, e principalmente a partir de 2012, a praça foi palco de manifestações políticas. O espaço público está adaptado para eventos cívicos, esportivos e feiras de exposições, além de receber um grande número de usuários que objetivam principalmente realizar atividades físicas, bem como pais que levam os filhos para brincar no parque infantil. 


\section{Revista Nacional de}

\section{RESULTADOS E DISCUSSÃO}

Neste capítulo, serão apresentados os resultados obtidos com a aplicação da metodologia por tópicos. As alterações mais significativas que ocorreram no período de estudo da praça, entre os anos de 2003 e 2017, foram no mobiliário e na vegetação.

\section{Estudo do mobiliário, estruturas e similares}

Em 2003, a praça apresentava um número significativamente menor de equipamentos quando comparada ao ano de 2017, principalmente aqueles voltados para o-lazer. Os mobiliários encontrados (Tabela 1) incluem bancos contínuos de concreto, luminárias de estilo republicano, lixeiras, entre outros.

Tabela 1. Levantamento quantitativo dos equipamentos e estruturas existentes na praça - Ano 2003

\begin{tabular}{|c|c|c|c|}
\hline EQUIPAMENTOS/ESTRUTURAS & SIM & NÃO & QUANTIDADE \\
\hline Bancos - material: concreto & $x$ & & -8 (168 metros) \\
\hline Mesa com bancos & $\mathrm{x}$ & & $\cdot 2$ (4 bancos cada) \\
\hline Iluminação (incandescente) & $x$ & & .30 \\
\hline Lixeiras & $\mathrm{x}$ & & .11 \\
\hline Sanitários & $x$ & & .2 \\
\hline Caminhos - material: bloco de concreto & $x$ & & $.2983 \mathrm{~m}^{2}$ \\
\hline Obra de arte - Monumento Rotary & $x$ & & 1 \\
\hline Espelho d'água/chafariz & & $x$ & \\
\hline Equipamentos para exercícios físicos & & $x$ & \\
\hline Parque infantil & & $x$ & \\
\hline Mastro para bandeira & & $x$ & \\
\hline
\end{tabular}

A avaliação qualitativa das estruturas encontradas na praça no ano de 2003 (Tabela 2) consideraram aspectos como o estado de conservação, disposição e design adequados. Os elementos não encontrados ou ainda não implantados na praça foram suprimidos das tabelas originais proposta pela metodologia. 
Tabela 2. Avaliação qualitativa da Praça Miguel Rossafa - Ano 2003

\begin{tabular}{lc}
\hline ESTRUTURAS AVALIADAS & NOTA \\
\hline Bancos & 3,4 \\
\hline Iluminação & 2,4 \\
\hline Lixeiras & 3,0 \\
\hline Sanitários & 2,4 \\
\hline Pisos & 4,0 \\
\hline Traçado dos caminhos & 3,8 \\
\hline Monumentos & 2,0 \\
\hline Vegetação & 1,4 \\
\hline Paisagismo & 0,3 \\
\hline Localização & 3,8 \\
\hline Conservação/limpeza & 3,8 \\
\hline Segurança & 2,3 \\
\hline Conforto Ambiental & 0,4 \\
\hline Pontuação: $0-\mid 0,5 \leftrightarrow p e ́$
\end{tabular}

Pontuação: $0-\mid$ 0,5 $\leftrightarrow$ péssimo; $0,5-\mid$ 1,5 $\leftrightarrow$ ruim; $1,5-\mid 2,5 \leftrightarrow$ regular; $2,5-\mid 3,5 \leftrightarrow$ bom; e 3,5-4,0 $\leftrightarrow$ ótimo Fonte: DE ANGELIS; CASTRO; ANGELIS NETO, 2004. Adaptado pelos autores.

Os bancos encontrados na praça são contínuos, feitos em concreto. Possuem 168 metros lineares distribuídos em oito bancos, sendo que um dos bancos previstos no projeto não foi construído. Os bancos não apresentam encosto, o que os tornam pouco ergonômicos. A iluminação é composta por luminárias baixas de estilo republicano, o que não representa o período no qual a praça foi reformada, tampouco representa a história da urbe, pois Umuarama é uma cidade fundada após o período republicano. As luminárias contêm lâmpadas incandescentes de baixa intensidade que resultam num ambiente pouco iluminado. Os sanitários não estão adaptados para pessoas portadoras de necessidades especiais, ainda que exista uma barra de apoio, porém está instalada incorretamente. Os pisos em blocos de concreto estão em bom estado, pois estão nivelados e apresentam um nível de atrito que impede o escorregamento. A paginação do piso foi realizada por meio dos recortes dos blocos de concreto e pela pintura colorida. O traçado dos caminhos está adequado ao trajeto percorrido pelos usuários. O monumento presente na praça é em homenagem aos 100 anos do Rotary International. Seu design é semelhante a um quadro em alvenaria, com desenhos em alto relevo apenas na face que está voltada para a rua, enquanto o outro lado é liso como uma parede. A disposição da homenagem dificulta a visualização da mesma, uma vez que está posicionada de modo a privilegiar a vista a partir dos veículos que trafegam na pista. $O$ paisagismo é praticamente inexistente. A vegetação é composta apenas por áreas gramadas e poucas árvores que não oferecem sombra de forma satisfatória, prejudicando o conforto térmico do espaço livre. A pontuação total da praça no ano de 2003 corresponde a 2,3 pontos, o que a classifica como regular.

No ano de 2017, a estrutura da praça foi alterada significativamente com a implantação de novos equipamentos, vegetação e paisagismo. As alterações incluem um parque infantil, a academia da terceira idade (ATI), plantio de árvores e forrações, entre outros (Tabela 3). Os itens que se mantiveram inalterados foram suprimidos da tabela. As modificações da praça 
alteraram as pontuações na avaliação qualitativa (Tabela 4), espeçialmente devido aos acréscimos de equipamentos na praça.

Tabela 3. Levantamento quantitativo dos equipamentos e estruturas existentes na praça - Ano 2017

\begin{tabular}{llll}
\hline EQUIPAMENTOS/ESTRUTURAS & SIM & NÃO & QUANTIDADE \\
\hline Mesa com bancos & & $\mathrm{x}$ & $\cdot$ \\
\hline lluminação (LED) & $\mathrm{x}$ & .30 \\
\hline Lixeiras & $\mathrm{x}$ & .12 \\
\hline Sanitários & $\mathrm{x}$ & .2 \\
\hline Caminhos - material: bloco de concreto & $\mathrm{x}$ & $.2983 \mathrm{~m}^{2}$ \\
\hline Obra de arte - Monumento Rotary e Estátua do Umuaraminha & $\mathrm{x}$ & 2 \\
\hline Espelho d'água/chafariz & $\mathrm{x}$ & 1 \\
\hline Equipamentos para exercícios físicos & $\mathrm{x}$ & $\mathrm{ATI}-10$ aparelhos \\
\hline Parque infantil & $\mathrm{x}$ & 1 \\
\hline Mastro para bandeira & $\mathrm{x}$ & 3 \\
\hline
\end{tabular}

Fonte: DE ANGELIS; CASTRO; ANGELIS NETO, 2004. Adaptado pelos autores.

Tabela 4. Avaliação qualitativa da Praça Miguel Rossafa - Ano 2017

\begin{tabular}{lc}
\hline ESTRUTURAS AVALIADAS & NOTA \\
\hline Bancos & 3,0 \\
\hline Iluminação & 3,5 \\
\hline Lixeiras & 2,5 \\
\hline Sanitários & 2,4 \\
\hline Pisos & 3,4 \\
\hline Traçado dos caminhos & 3,8 \\
\hline Monumentos & 2,2 \\
\hline Vegetação & 2,4 \\
\hline Paisagismo & 1,5 \\
\hline Localização & 3,8 \\
\hline Conservação/limpeza & 3,0 \\
\hline Segurança & 3,4 \\
\hline Conforto Ambiental & 2,0 \\
\hline Espelho d'água/chafariz & 2,4 \\
\hline Equipamentos para exercícios físicos & 1,4 \\
\hline Parque infantil & 2,6 \\
\hline Ponçã $0-\mid 0,5 \leftarrow$ pessimo; 0,5 $11,5 \leftrightarrow r u m$
\end{tabular}

Pontuação: $0-\mid 0,5 \leftrightarrow$ péssimo; $0,5-\mid 1,5 \leftrightarrow$ ruim; $1,5-\mid 2,5 \leftrightarrow$ regular; $2,5-\mid 3,5 \leftrightarrow$ bom; e 3,5-4,0 $\leftrightarrow$ ótimo Fonte: DE ANGELIS; CASTRO; ANGELIS NETO, 2004. Adaptado pelos autores.

Os bancos de concreto precisam de manutenção na pintura. Um dos bancos foi modificado pelos usuários da praça (teve uma cantoneira metálica instalada para a prática de skate). As mesas com bancos, presentes na praça em 2003, foram removidos pois estavam quebrados. A iluminação foi inteiramente substituída por luminárias de design moderno com lâmpadas de LED branca, que aumentaram a intensidade de luz emitida para o ambiente. Entretanto, a parte central da praça não recebe iluminação suficiente, permanecendo escura. Uma lixeira para lixos recicláveis foi incorporada à praça, porém algumas das lixeiras antigas estão quebradas, necessitando de manutenção. Os sanitários, principalmente o masculino, precisa de limpeza. As paredes externas dos sanitários receberam desenhos em pintura em ambos os lados (um cachorro e o rosto de uma mulher), que deixaram o ambiente mais alegre. Os pisos 

Tabela 5. Levantamento quantitativo da vegetação - Ano 2003

\begin{tabular}{|c|c|c|c|c|c|c|c|}
\hline \multirow{2}{*}{ Cód* } & \multirow{2}{*}{$\begin{array}{l}\text { Nome } \\
\text { comum }\end{array}$} & \multirow{2}{*}{ Nome científico } & \multirow{2}{*}{ Família } & \multirow{2}{*}{$\begin{array}{l}\text { № de } \\
\text { indivíduos }\end{array}$} & \multirow{2}{*}{$\begin{array}{l}\text { Frequência } \\
\text { relativa (\%) }\end{array}$} & \multicolumn{2}{|l|}{ Folha } \\
\hline & & & & & & Caduca & Perene \\
\hline AV & Ficus & Ficus benjamina & Moraceae & 3 & 27,3 & & $x$ \\
\hline AV & Oiti & $\begin{array}{l}\text { Licania } \\
\text { tomentosa }\end{array}$ & Chrysobalanaceae & 5 & 45,4 & & $x$ \\
\hline PA & $\begin{array}{l}\text { Palmeira } \\
\text { imperial }\end{array}$ & $\begin{array}{l}\text { Roystonea } \\
\text { olerace }\end{array}$ & Palmae & 2 & 18,2 & & $x$ \\
\hline $\mathrm{PA}$ & Jerivá & $\begin{array}{l}\text { Syagrus } \\
\text { romanzoffiana }\end{array}$ & Arecaceae & 1 & 9,1 & & $x$ \\
\hline
\end{tabular}

Código: AV: árvore; PA: palmácea. Fonte: DE ANGELIS; CASTRO; ANGELIS NETO, 2004. Modificado pelos autores.

Durante os anos, novas espécies arbóreas e herbáceas foram incorporadas à vegetação da praça (Tabela 6). Uma vez que a vegetação herbácea (forração) é substituída quando as plantas perdem sua vitalidade, as espécies utilizadas variam de acordo com a disponibilidade de mudas no viveiro. Portanto, estas espécies não foram identificadas.

Tabela 6. Levantamento quantitativo da vegetação - Ano 2017

\begin{tabular}{|c|c|c|c|c|c|c|c|}
\hline \multirow{2}{*}{ Cód* } & \multirow{2}{*}{$\begin{array}{l}\text { Nome } \\
\text { comum }\end{array}$} & \multirow{2}{*}{ Nome científico } & \multirow{2}{*}{ Família } & \multirow{2}{*}{$\begin{array}{c}\text { № de } \\
\text { indivíduos }\end{array}$} & \multirow{2}{*}{$\begin{array}{l}\text { Frequência } \\
\text { relativa (\%) }\end{array}$} & \multicolumn{2}{|c|}{ Folha } \\
\hline & & & & & & Caduca & Perene \\
\hline AV & Ficus & Ficus benjamina & Moraceae & 3 & 13,6 & & $x$ \\
\hline AV & Oiti & $\begin{array}{l}\text { Licania } \\
\text { tomentosa }\end{array}$ & Chrysobalanaceae & 5 & 22,8 & & $x$ \\
\hline AV & $\begin{array}{l}\text { Coreutéria ou } \\
\text { Árvore-da- } \\
\text { China }\end{array}$ & $\begin{array}{l}\text { Koelreuteria } \\
\text { paniculata laxm }\end{array}$ & Sapindaceae & 1 & 4,5 & $x$ & \\
\hline AV & Ipê rosa & $\begin{array}{l}\text { Handroanthus } \\
\text { heptaphyllus }\end{array}$ & Bignoniaceae & 2 & 9,1 & $x$ & \\
\hline AV & Ipê amarelo & Tabebuia alba & Bignoniaceae & 8 & 36,4 & $x$ & \\
\hline PA & $\begin{array}{l}\text { Palmeira } \\
\text { imperial }\end{array}$ & $\begin{array}{l}\text { Roystonea } \\
\text { olerace }\end{array}$ & Palmae & 2 & 9,1 & & $x$ \\
\hline PA & $\begin{array}{l}\text { Coqueiro } \\
\text { Jerivá }\end{array}$ & $\begin{array}{l}\text { Syagrus } \\
\text { romanzoffiana }\end{array}$ & Arecaceae & 1 & 4,5 & & $x$ \\
\hline
\end{tabular}

Código: AV: árvore; PA: palmácea. Fonte: De Angelis; Castro; Angelis Neto, 2004. Modificado pelos autores.

As árvores incorporadas aumentaram a variedade de espécies, porém, ainda existe uma concentração maior de uma delas, a Tabebuia alba. A espécie Licania tormentosa, que apresentava o maior número de indivíduos em 2003, agora apresenta a segunda maior frequência relativa. A escolha do local de plantio das novas árvores não foi planejada de modo a obter sombreamento nas áreas de permanência de usuários (bancos). Assim, ainda que o número de indivíduos vegetais tenha aumentado, estes ainda são insuficientes para promover o conforto térmico ao espaço livre, em especial pelo porte das árvores, que se encontram ainda em desenvolvimento. 



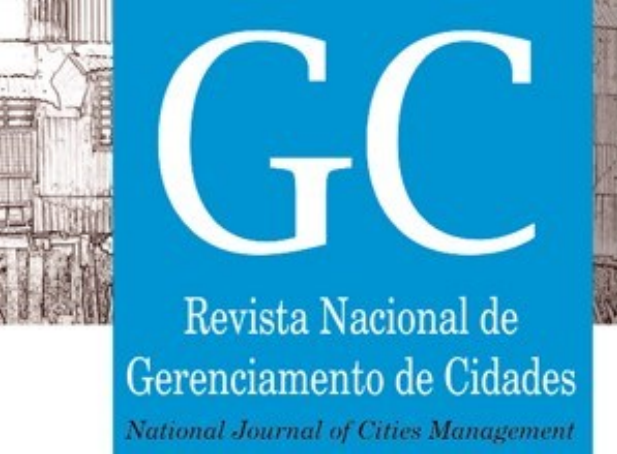

\section{AGRADECIMENTO}

Agradecemos a CAPES (Coordenação de Aperfeiçoamento de Pessoal de Nível Superior) pelo apoio financeiro.

\section{REFERÊNCIAS}

CAVALHEIRO, Felisberto; DEL PICCHIA, Paulo Celso Dornelles. Áreas verdes: conceitos, objetivos $e$ diretrizes para o planejamento. In: Encontro Nacional Sobre Arborização Urbana, 4 Vitória-ES, de 13 a 18 de set/92. Anais I e II, 1992, p. 29-38.

DE ANGELIS, Bruno Luiz Domingos; CASTRO, Rosana Miranda de; ANGELIS NETO, Generoso De. Metodologia para levantamento, cadastramento, diagnóstico e avaliação de praças no Brasil. Revista de Engenharia Civil. Universidade do Minho. Braga, n. 20, p. 57-70, 2004.

GALENDER, Fany Cutcher. A Idéia de Sistema de Espaços Livres Públicos na Ação de Paisagistas Pioneiros na América Latina. Paisagens Em Debate, n. 03, São Paulo, nov. 2005.

GOMES, Marcos Antônio Silvestre. De Largo A Jardim: Praças Públicas No Brasil - Algumas aproximações. Estudos Geográficos, Rio Claro, n. 5, p. 101-120, 2007.

HULSMEYER, Alexander Fabbri. Qualidade do Espaço Livre Urbano: Valores ambientais, culturais e funcionais. 234 f. Dissertação (Mestrado em Geografia Meio Ambiente e Desenvolvimento) Universidade Estadual de Londrina, Londrina, 2004.

MAGNOLI, Miranda M. E. M. Espaços livres e urbanização: Uma introdução a aspectos da paisagem metropolitana. 1982. Tese (Livre-docência) - Faculdade de Arquitetura e Urbanismo, Universidade de São Paulo, São Paulo, 1982.

NUCCl, João Carlos. Qualidade ambiental e adensamento urbano: um estudo de ecologia e planejamento da paisagem aplicado ao distrito de Santa Cecília (MSP). 2a ed. Curitiba: O Autor, 2008. $150 \mathrm{p}$.

PREFEITURA MUNICIPAL DE UMUARAMA. Projeto Paisagístico da requalificação da Praça Miguel Rossafa, 2003. Disponibilizado em arquivo digital formato dwg.

ROBBA, Fábio; MACEDO, Silvio Soares. Praças Brasileiras = Public Squares in Brazil. 3 ed. (1 ed - 2002). Edusp - Editora da Universidade de São Paulo: São Paulo, 2010. [Coleção Quapá].

SITTE, Camillo. A Construção de Cidades Segundo Princípios Artísticos. Trad. Ricardo Ferreira Henrique. São Paulo: Ática, 1992.

UMUARAMA. Lei Ordinária no 2275, de 04 de maio de 2000. Autoriza o Poder Executivo a readequar a Praça Miguel Rossafa. 